\title{
Vortex molecules in thin films of layered superconductors
}

\author{
A. V. Samokhvalov ${ }^{\text {a) }}$ and A. S. Mel'nikov \\ Institute for Physics of Microstructures, Russian Academy of Sciences, GSP 105, Nizhny Novgorod 603950, \\ Russia and N.I. Lobachevsky State University of Nizhny Novgorod, Nizhny Novgorod 603950, Russia
}

\section{A. I. Buzdin}

University of Bordeaux, LOMA UMR CNRS 5798, F 33405 Talence Cedex, France and Department of Materials Science and Metallurgy, University of Cambridge, CB3 OFS Cambridge, United Kingdom

\begin{abstract}
The seminal work of Abrikosov on the vortex lattices in type-II superconductors has triggered the creation and rapid development of the whole new field of condensed matter physics, namely, the physics of vortex matter in a large variety of superfluid and superconducting systems. Among these systems and compounds the layered superconductors are known to form a very important class which is associated with a number of fascinating new discoveries in vortex physics. The phenomenon of the vortex attraction in tilted magnetic fields provides one of the examples of such findings which affected theoretical and experimental research in the field for almost two decades. In our paper we review some recent advances in this direction focusing on the intervortex interaction and equilibrium vortex structures in thin films of layered superconductors in magnetic field tilted with respect to the layers. In such a case the magnetic field penetrates superconductor in the form of tilted vortices or a crossing array of Josephson vortices and pancake stacks. We study the interplay between two different long-range potentials: (i) attraction of tilted vortices or deformed stacks; (ii) the Pearl's repulsion. This interplay is responsible for the formation of the minimum in the total interaction energy and resulting decay of vortex chains in clusters. The number of Abrikosov vortices in these clusters (or vortex molecules) depends on field tilting angle and film thickness.
\end{abstract}

\section{Introduction}

Abrikosov contributed to many domains of theoretical physics: condensed matter, plasma physics, physics of high pressure, and quantum electrodynamics. However, the Nobel Prize in Physics 2003 was awarded to Abrikosov for his theoretical discovery of vortices and the theory of type-II superconductivity. These vortices are now often called Abrikosov vortices and the understanding of the properties of the mixed state of type-II superconductors is ultimately related to the paradigm of "vortex matter." Thermal excitations, vortex pinning, crystal anisotropy, spatial and time dependent magnetic field, all these reveal panoply of different transitions in the vortex matter, which makes its physics very rich. ${ }^{1}$

Both the equilibrium and transport properties of the vortex matter are essentially affected by the behavior of the potential of the interaction between vortices. In isotropic bulk superconductors the intervortex interaction potential is well known to be always repulsive ${ }^{2}$ and screened at intervortex distances $R$ greater than the London penetration depth $\lambda$. As a result, in perfect crystals quantized Abrikosov vortices (vortex lines) form a triangular flux lattice. ${ }^{3}$ The magnetic flux through the unit cell of such flux line lattice equals to the flux quantum $\varphi_{0}=\pi \hbar c / e$, and each unit cell is occupied by the single vortex.

This textbook picture is known to change drastically in layered superconductors in tilted magnetic field due to the phenomenon of the long-range attraction between tilted ${ }^{4-8}$ or deformed $^{9,10}$ vortex lines composed of two dimensional 2D pancake vortices (PVs). For bulk layered superconductors with a moderate anisotropy (like $\mathrm{NbSe}_{2}, \mathrm{YBaCuO}$ ), the low magnetic field penetrates mainly in form of the tilted vortex lines which are arranged in chains [Fig. 1(a)]. These vortex chains have been observed experimentally by the decoration technique in $\mathrm{YBa}_{2} \mathrm{Cu}_{3} \mathrm{O}_{7},{ }^{11}$ scanning-tunneling microscopy in $\mathrm{NbSe}_{2}{ }^{12}$ and Lorentz microscopy measurements in $\mathrm{YBa}_{2} \mathrm{Cu}_{3} \mathrm{O}_{7}$ (Ref. 13) (see Ref. 14 for a review). For strongly anisotropic superconductors like $\mathrm{Bi}_{2} \mathrm{Sr}_{2} \mathrm{CaCu}_{2} \mathrm{O}_{8+\delta}$ (BSCCO) the chain consists of a lattice of in-plane Josephson vortices (JVs) crossing with pancake stacks perpendicular to the planes [Figs. 1(b) and 1(c)]. The attraction between $\mathrm{PVs}$ and $\mathrm{JVs}^{15}$ leads to the deformation of the pancake stacks detected by the modern vortex imaging methods such as Lorentz microscopy, ${ }^{13}$ high-resolution scanning Hall probe, ${ }^{17}$ Bitter decoration techniques, ${ }^{18}$ and magneto-optical measurements ${ }^{19}$ (see also Ref. 14 as a review). A lot of other groundstate vortex chain configurations and phase transitions between them were studied in detail in Ref. 20. The potential of the interaction between vortices in layered superconductors depends on the internal structure of vortex lines and is determined by two different kinds of interaction between pancake vortices in different layers: magnetic and Josephson interactions. The relative strength of these two interactions depends on the anisotropy parameter $\Gamma=\lambda_{c} / \lambda_{a b}$ and interlayer spacing $s$, where $\lambda_{c}$ is the penetration depth for currents along the $c$ axis and $\lambda_{a b}$ is the penetration depth for currents in the $a b$ plane (parallel to the layers). The distinctive feature of anisotropic superconductors is known to be an unusual attractive part of the vortex vortex interaction potential in tilted magnetic field. ${ }^{4-10}$ Tilted or deformed PVs stacks attract each other in the plane defined by the anisotropy axes 

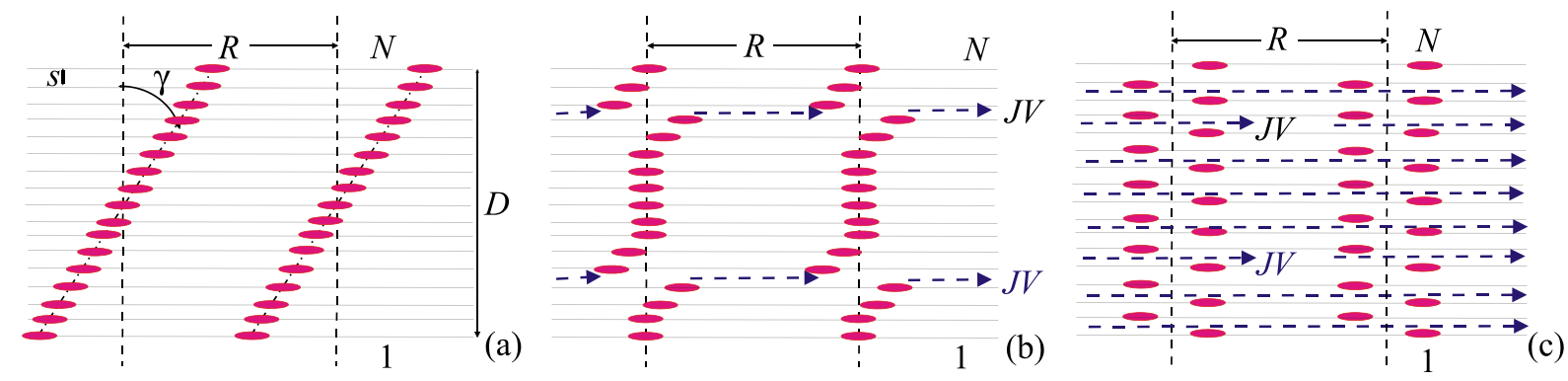

Fig. 1. Schematic representation of a layered superconductor with tilted (a) and crossing (b) and (c) vortex structures, $s$ is a distance between the layers, $N$ is the number of layers, $D \quad\left(\begin{array}{ll}N & 1\end{array}\right) s$ is the total thickness of the structure.

and vortex line, and the value of the attractive force is controlled by the tilting angle of the vortex line with respect to the anisotropy axis. Certainly, the interaction between vortex stacks at small distances $R \sim \lambda$ remains repulsive. Interestingly, the attractive long-range intervortex potential has the same asymptotic behavior $U_{a} \sim-1 / R^{2}(R \gg \lambda)$ for both cases: for tilted vortex lines ${ }^{4-8}$ and for deformed stacks. ${ }^{9,10}$ Note, that the attractive interaction between any two vortices in the chain (except the nearest neighbors) provides the stability of the whole vortex chain.

In thin films of layered superconductors, however, such van der Waals type potential appears to be strongly modified by the long-ranged Pearl's repulsion ${ }^{21}$ which could mask the subtle attraction of vortices. The Pearl's potential $U_{P}(r)$ of intervortex interaction is always repulsive, decays extremely slowly $\left(U_{P} \sim 1 / R\right.$ for $\left.R>\lambda\right)$ and always dominates at large distances $R>R_{m} \gg \lambda$. It means that the repulsion between two vortices is restored at intervortex distance $R>R_{m}$ and stability of the vortex chain of a length $L \gtrsim R_{m}$ is destroyed. As a result, the formation of an infinite vortex chain becomes unfavorable. Adding vortices one by one we can find an optimal number of vortices which can be arranged in a chain of a finite length. As a result, there appears an intriguing possibility to form a vortex structure consisting of finite size chains, i.e., of vortex molecules. By varying either the film thickness or the tilting angle we can modify the balance between the attractive and repulsive interactions, which should determine the size of the energetically favorable vortex configurations.

Modern vortex imaging techniques provide a possibility to observe the crossover between different intervortex interaction regimes in thin film samples. In particular, the magnetic field distributions induced by vortices in thin films can be probed by the penetrating electron beam used in Lorentz microscopy measurements. ${ }^{22,23}$ This technique, owing to the low penetration power of the existing $300-\mathrm{kV}$ field-emission beam, permits us to work with films of thicknesses smaller than $0.51 \mu \mathrm{m}$. It is, therefore, "par excellence" an ideal tool to study the peculiarities of the vortex structures in thin films. In Ref. 24 it was demonstrated how the Lorentz microscopy technique permits to discover the special characters of the intervortex interaction in $\mathrm{YBaCuO}$ films of thickness $d \simeq 0.5$ $\mu \mathrm{m}$, placed in a tilted magnetic field. The vortex structure changes qualitatively for a fixed external magnetic field $\mathbf{B}_{0}$ direction by increasing absolute value of the field $B_{0}$. The increase in the field value $B_{0}$ causes an increase in the tilting angles of vortex lines. Therefore, the vortex attraction prevails at higher $B_{0}$ values, while at low fields the attraction force is overcome by the repulsive force due to Pearl's effect, and the vortex chains are expected to disappear. Indeed, such a qualitative change in the vortex structure is confirmed ${ }^{24}$ by the experimental data: at low fields, which correspond to small tilting values, the vortex chains are completely absent, while at rather high fields $B_{0}>B^{*}$, where $B^{*}$ is the critical field, the formation of vortex chains is clearly observed.

In this paper we focus on the calculations of the pair potential of the intervortex interaction in thin films of layered superconductors. Our calculations are based on the Lowrence Doniach theory ${ }^{25}$ which is simplified neglecting the effects of weak interlayer Josephson coupling. This approximation of Josephson-decoupled superconducting (SC) layers ${ }^{26}$ is known to be useful in studies of the vortex lattice structure for very weak coupling of the layers $\lambda_{J}=\Gamma_{s} \gg \lambda_{a b}$, where the Josephson length $\lambda_{J}=\Gamma_{s}$ is the in-plane size of the Josephson core radius. $^{27}$ The equilibrium shape of a single vortex line depends on the in-plane magnetic field $\mathbf{B}_{\|}=B_{\|} \mathbf{x}_{0}$ distribution and can be very different. Without the in-plane field, the pancakes form a vertical stack. If the in-plane field $\mathbf{B}_{\|}$is rather small $\left(B_{\|} \ll H_{0}=\varphi_{0} / \Gamma s^{2}\right)$ and does not penetrate inside superconductor in form of Josephson vortices, then screening Meissner currents tilt and bend the vortex line. Bending of the vortex line is essential only near the surface of the SC film and often can be neglected. ${ }^{28}$ In Sec. 2.2 we study the interaction between two straight stacks of PVs tilted at a certain angle $\gamma$ with respect to the c direction, as shown in Fig. 1(a). In fact in layered superconductors with very weak interlayer coupling the Josephson vortices will appear at much lower field. As a result, at tilted magnetic field, crossing lattice of pancakes, formed by Abrikosov vortices, and Josephson vortices is more energetically preferable than a lattice of tilted vortex stacks. ${ }^{15-17}$ The interaction between pancakes and in-plane field in the form of Josephson vortices produces zigzag deformation of the stack of the pancakes. ${ }^{30}$ This deformation is responsible for a long-range attraction between such stacks ${ }^{9}$ which is quite similar to the case of tilted vortex lines. In Sec. 2.3 we study the extreme case when the in-plane magnetic field $B_{\|} \gg H_{0}$ creates the dense triangular lattice of JVs which are strongly compressed along the $c$ axis due to a very high anisotropy $\Gamma \gg 1$. This dense triangular lattice of JVs produces zigzag displacement of $\mathrm{PVs}^{30}$ along the $x$ axis, so that the pancake centers for each vortex line are positioned along the broken line [see Fig. 1(c)]. We calculate the interaction potential between two zigzag lines in a thin SC film. In Sec. 2.4 we compare these two extreme cases and discuss qualitatively the condition of the vortex attraction if the in-plane magnetic field is relatively small $\left(B_{\|} \sim H_{0}\right)$ and the crossing points between 
JVs and PVs are rare. In Sec. 3 we discuss the condition of vortex cluster formation and phase transitions between the vortex lattices with different number of vortices per unit cell.

\section{Interaction potential for two stacks of pancakes}

As a model of a film of strongly anisotropic superconductor we consider a finite set of $N$ thin SC layers with the thickness $d$ much smaller $\lambda$ located at a distance $s$ from each other, so that the $n$th SC layer coincides with the plane $z=z_{n}=n s(1 \leq n \leq N)$. The field component perpendicular to the layers creates the vortex line which pierces the film as a stack of PVs centered at the points $\mathbf{r}_{n}=x_{n} \mathbf{x}_{0}+y_{n} \mathbf{y}_{0}$ in the $n$th layer. We derive general expressions for interaction potential of two identical stacks of PVs taking into account both long-range attraction and repulsion phenomena. We study both the limits of weak $\left(B_{\|} \ll H_{0}\right)$ and strong $\left(B_{\|}\right.$ $\left.\gg H_{0}\right)$ in-plane magnetic field. The shape of the interacting vortex lines is assumed to be fixed and not affected by the vortex vortex interaction potential. Certainly, such assumption is valid only in the limit of rather larger distances between the vortex lines when the effect of interaction on the vortex shape can be viewed as a small perturbation.

\subsection{Basic equations}

Within the model of the set of Josephson-decoupled SC layers, pancakes interact with each other only via magnetic fields. General equation for the vector potential A distribution in such system reads

$$
\operatorname{rot} \operatorname{not} \mathbf{A}=\frac{4 \pi}{c} \sum_{n, m=1}^{N} \mathbf{J}_{n}^{m}(\mathbf{r}) \delta\left(z-z_{n}\right) .
$$

The sheet current at the $n$th layer created by the pancake at $m$ th layer takes the form

$$
\mathbf{J}_{n}^{m}(\mathbf{r})=\frac{c}{4 \pi \Lambda}\left[\mathbf{\Phi}\left(\mathbf{r}-\mathbf{r}_{m}\right) \delta_{n m}-\mathbf{A}^{m}\left(\mathbf{r}, z_{n}\right)\right],
$$

where $\Lambda=\lambda^{2} / d=\lambda_{a b}^{2} / s$ is the effective penetration depth in a superconducting film, $\mathbf{A}^{m}(\mathbf{r}, z)$ is the vector potential induced by the only pancake vortex located in the $m$ th layer.

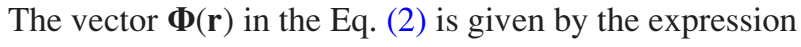

$$
\boldsymbol{\Phi}(\mathbf{r})=\frac{\varphi_{0}}{2 \pi} \frac{\left[\mathbf{z}_{0} \times \mathbf{r}\right]}{\mathbf{r}^{2}}
$$

For the layered system without Josephson coupling a general expression for the free energy can be written in the form

$$
F=\frac{1}{8 \pi} \int d V\left[\left(\operatorname{rot} \mathbf{A}^{2}\right)+\left(\frac{4 \pi}{c}\right)^{2} \Lambda \sum_{n=1}^{N} \mathbf{J}_{n}^{2}(\mathbf{r}) \delta\left(z-z_{n}\right)\right]
$$

The total vector potential $\mathbf{A}(\mathbf{r}, z)$ and the sheet current in the $n$th layer $\mathbf{J}_{n}(\mathbf{r})$, produced by an arbitrary vortex line are the sum of the contributions induced by $N$ pancakes:

$$
\mathbf{A}(\mathbf{r}, z)=\sum_{m=1}^{N} \mathbf{A}^{m}(\mathbf{r}, z), \quad \mathbf{J}_{n}(\mathbf{r})=\sum_{m=1}^{N} \mathbf{J}_{n}^{m}(\mathbf{r}),
$$

and can by found from Eqs. (1) (3) using an approach similar to that proposed in Refs. 32 and 33 (see Ref. 28 for details).
Using the gauge div $\mathbf{A}=0$ and the Fourier transforms

$$
\begin{gathered}
\mathbf{A}(\mathbf{q}, k)=\int d^{2} \mathbf{r} d z \mathrm{e}^{i \mathbf{q r}+i k z} \mathbf{A}(\mathbf{r}, z) \\
\mathbf{A}_{n}(\mathbf{q})=\int d^{2} \mathbf{r} \mathrm{e}^{i \mathbf{q r}} \mathbf{A}\left(\mathbf{r}, z_{n}\right), \quad \mathbf{J}_{n}(\mathbf{q})=\int d^{2} \mathbf{r} \mathrm{e}^{i \mathbf{q r}} \mathbf{J}_{n}(\mathbf{r})
\end{gathered}
$$

one can rewrite the basic Eq. (1) for each vortex line in the momentum representation as follows:

$$
\left(q^{2}+k^{2}\right) \mathbf{A}(\mathbf{q}, k)=\frac{1}{\Lambda} \sum_{n}\left[\boldsymbol{\Phi}_{n}(\mathbf{q})-\mathbf{A}_{n}(\mathbf{q})\right] \mathrm{e}^{i k n s},
$$

where

$$
\boldsymbol{\Phi}_{n}(\mathbf{q})=\boldsymbol{\Phi}(\mathbf{q}) \mathrm{e}^{i \mathbf{q} \mathbf{r}_{n}}, \quad \boldsymbol{\Phi}(\mathbf{q})=-i \varphi_{0} \frac{\left[\mathbf{z}_{0} \times \mathbf{q}\right]}{q^{2}} .
$$

These equations can be reduced to the scalar form

$$
f_{n}+\frac{1}{2 q \Lambda} \sum_{m} \mathrm{e}^{|n m| q s} f_{m}=\mathrm{e}^{i q \mathbf{r}_{n}}
$$

where we introduce the new functions $f_{n}(\mathbf{q})$ :

$$
\mathbf{J}_{n}(\mathbf{q})=\frac{c}{4 \pi \Lambda}\left(\boldsymbol{\Phi}_{n}(\mathbf{q})-\mathbf{A}_{n}(\mathbf{q})\right)=\frac{c}{4 \pi \Lambda} \boldsymbol{\Phi}(\mathbf{q}) f_{n}(\mathbf{q}) .
$$

The solution of the linear system (8) for a fixed configuration of pancakes $\mathbf{r}_{n}$ determines the distribution of the vector potential $\mathbf{A}(\mathbf{r}, z)$ which is created by an arbitrary vortex line in a finite stack of superconducting layers.

For two vortex lines we can write the total vector potential and the total sheet current as superpositions of contributions coming from the first $\left(\mathbf{A}_{n}^{(1)}, \mathbf{J}_{n}^{(1)}\right)$ and second $\left(\mathbf{A}_{n}^{(2)}, \mathbf{J}_{n}^{(2)}\right)$ vortices. Calculating the interaction energy $\varepsilon_{\text {int }}$ of vortex lines we should keep in the free energy only the terms which contain the products of fields corresponding to different vortex lines. As a result, in the momentum representation the general expression (4) for the free energy of the layered system without Josephson coupling reads

$$
\begin{aligned}
\varepsilon_{\text {int }}= & \frac{1}{32 \pi^{3} \Lambda} \sum_{n} \int d^{2} \mathbf{q}\left[\left(\boldsymbol{\Phi}_{n}^{(1)}(\mathbf{q})-\mathbf{A}_{n}^{(1)}(\mathbf{q})\right) \boldsymbol{\Phi}_{n}^{(2)}(-\mathbf{q})\right. \\
& \left.+\left(\boldsymbol{\Phi}_{n}^{(2)}(\mathbf{q})-\mathbf{A}_{n}^{(2)}(\mathbf{q})\right) \mathbf{\Phi}_{n}^{(1)}(-\mathbf{q})\right] .
\end{aligned}
$$

For the particular case of two identical (parallel) vortex lines which are shifted at the vector $\mathbf{R}=\mathbf{r}_{n}^{(2)}-\mathbf{r}_{n}^{(1)}(n=1$ $-N)$ in the $x y$ plane we get following general expression for the interaction energy via the scalar functions $f_{n}(\mathbf{q}):{ }^{28}$

$$
\varepsilon_{\text {int }}(\mathbf{R})=\frac{\varphi_{0}^{2}}{16 \pi^{3} \Lambda} \int \frac{d^{2} \mathbf{q}}{q} \cos (\mathbf{q} \mathbf{R}) \sum_{n} f_{n}(\mathbf{q}) e^{i \mathbf{q r}_{n}} .
$$

The expression (11) and Eq. (8) determine the interaction energy of two identical vortex lines.

\subsection{Interaction potential of tilted stacks of pancakes}

In the limit of weak in-plane magnetic field $\left(B_{\|} \ll H_{0}\right)$ we neglect the vortex line bending and restrict ourselves to the case of straight vortex lines parallel to the plane $x y$ and tilted at a certain angle $\gamma$ with respect to the anisotropy axis 
c ( $z$ axis) as shown in Fig. 1(a). Let us evaluate the interaction energy (11) of two tilted parallel vortex lines taking

$$
\mathbf{r}_{n}^{(1)}=n s \tan \gamma \mathbf{x}_{0}, \quad \mathbf{r}_{n}^{(2)}=\mathbf{r}_{n}^{(1)}+\mathbf{R}
$$

For the case of a large intervortex distance $R \gg s$, the solution of the problem (8) may be simplified, because for the relevant wave vectors $q \sim 1 / R$ the conditions of the continuous limit are satisfied: $q s \ll 1$ and $q_{x} s \tan \gamma \ll 1$. Introducing a continuous coordinate $z=n s$ and continuous function $f_{\mathbf{q}}$ $(z)$, the linear system of Eq. (8) reduces to the following integral equation

$$
f_{\mathbf{q}}(z)+\frac{1}{2 q \lambda_{a b}^{2}} \int_{D / 2}^{D / 2} d z^{\prime} \mathrm{e}^{q\left|z z^{\prime}\right|} f_{\mathbf{q}}\left(z^{\prime}\right)=\mathrm{e}^{i q_{x} z \tan \gamma}
$$

Equation (12) can be rewritten as a differential one

$$
\frac{d^{2} f_{\mathbf{q}}}{d z^{2}}-\left(\lambda_{a b}^{2}+q^{2}\right) f_{q}(z)=-\left(q_{x}^{2} \tan ^{2} \gamma+q^{2}\right) \mathrm{e}^{i q_{x} z \tan \gamma}
$$

at the interval $-D / 2<z<D / 2$ with the boundary conditions

$$
\left.\left(\frac{d f_{\mathbf{q}}}{d z} \pm q f_{\mathbf{q}}\right)\right|_{ \pm D / 2}=\left(i q_{x} \tan \gamma \pm q\right) \mathrm{e}^{ \pm i q_{x} D \tan \gamma / 2}
$$

where $D=(N-1) s$ is the thickness of superconducting film. Skipping the details of the solution of Eqs. (13) and (14), we get the following expression for the interaction energy (11) in the continuous limit: ${ }^{28}$

$$
\varepsilon_{\mathrm{int}}=\frac{\varphi_{0}^{2}}{16 \pi^{3} \lambda_{a b}^{2}} \int \frac{d^{2} \mathbf{q}}{q^{2}} \cos (\mathbf{q} \mathbf{R}) S(\mathbf{q})
$$

where the expression for the kernel $S(\mathbf{q})$ takes form

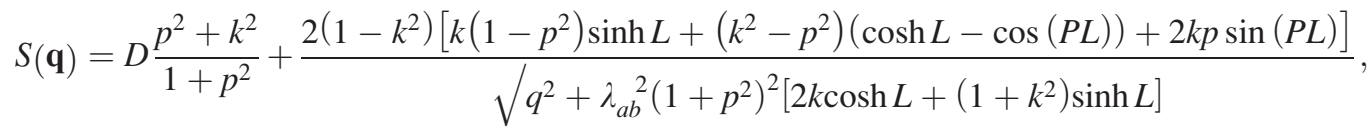

$$
\begin{aligned}
& L=D \sqrt{ } q^{2}+\lambda_{a b}{ }^{2}, \quad k=q / \sqrt{ } q^{2}+\lambda_{a b}{ }^{2}, \quad p=q_{x} \tan \gamma / \sqrt{ } q^{2}+\lambda_{a b}{ }^{2} .
\end{aligned}
$$

The first term in the kernel (16) describes the intervortex interaction in the bulk system, while the second term is responsible for the Pearl effect due to film boundaries.

The minimum energy configuration corresponds to the case $R_{y}=0$. In Fig. 2 we present some typical plots of the interaction energy $\varepsilon_{\text {int }}\left(R_{x}, R_{y}=0\right)$ vs the distance $R_{x}=R$ for $D=3 \lambda_{a b}$ which corresponds to the Lorentz microscopy experiments in $\mathrm{YBCO}^{24}$ and $\mathrm{Bi}-2212^{13}$ samples. Analyzing the dependence $\varepsilon_{\text {int }}(R)$, one can separate three contributions to the energy of vortex vortex interaction: (i) a short-range repulsion which decays exponentially with increasing intervortex distance $R$ (for $R>\lambda_{a b}$ ); (ii) an intervortex attraction which is known to be specific for tilted vortices in anisotropic systems;

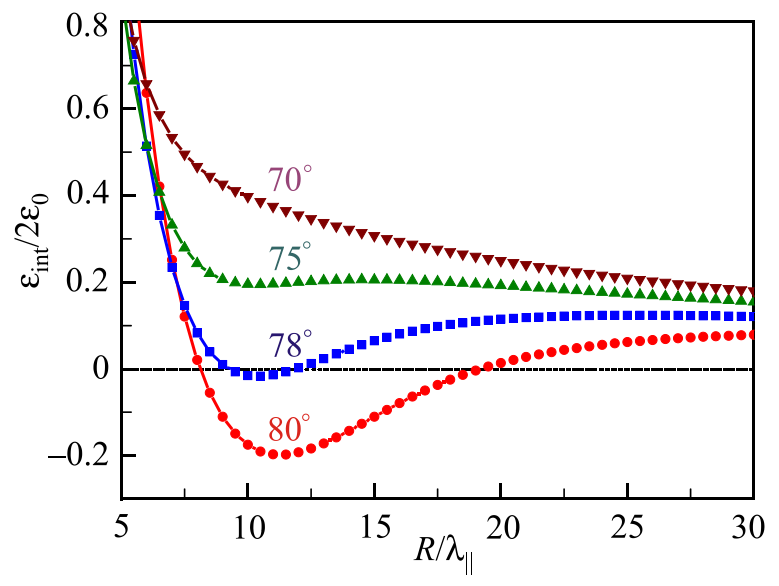

Fig. 2. Typical plots of the interaction energy per vortex [Eqs. (15) and (16)] vs the distance $R$ between two vortices for a film of thickness $D \quad 3 \lambda_{a b}$ and different tilting angles $\gamma \quad 70^{\circ}, 75^{\circ}, 78^{\circ}, 80^{\circ}\left(\varepsilon_{0} \quad \varphi_{0}^{2} / 16 \pi^{3} \lambda_{a b}\right)$. this attraction energy term decays as $R^{2}$ and strongly depends on the angle $\gamma$ between the vortex axis and the c direction; (iii) long-range (Pearl) repulsion which decays as $R^{1}$ and results from the surface contribution to the energy. Note that the third term does exist even for a large sample thickness $D$ (see Ref. 29) although in the limit $D \gg \lambda_{a b}$ it is certainly masked by the dominant bulk contribution. At $R \gg \lambda_{a b}$ the short-range interaction term vanishes and the interaction energy vs $R$ takes the simple form

$$
\varepsilon_{\text {int }} \simeq \frac{\varphi_{0}^{2}}{8 \pi^{2}}\left(-\frac{D_{\text {eff }} \tan ^{2} \gamma}{R^{2}}+\frac{2}{R}\right),
$$

where $D_{\text {eff }}=D-2 \lambda_{a b} \tanh \left(D / 2 \lambda_{a b}\right)$ is the effective film thickness. One can observe here an interplay between the long-range attractive [first term in Eq. (17)] and the longrange repulsive [second term in Eq. (17)] forces. Note that the $\lambda_{a b}$ value increases with an increase in temperature, thus, the effective thickness decreases and the long-range attraction force appears to be suppressed with increasing temperature. For large $R$ the energy is always positive and corresponds to the vortex repulsion similar to the one between the pancakes in a single layer system. With a decrease in the distance $R$ the attraction force comes into play resulting in the change of the sign of the energy. Such behavior points to the appearance of the minimum in the interaction potential.

\subsection{Interaction potential of zigzag stacks of pancakes}

In the limit of strong in-plane magnetic field $\left(B_{\|} \gg H_{0}\right)$ the tilted magnetic field penetrates in the form of the crossing lattice comprising the in-plane Josephson vortices and per- 
high-field limit, $B_{\|}>H_{0}=\varphi_{0} / s \lambda_{J}$, all interlayer junctions are homogeneously filled with Josephson vortices, which form the dense triangular lattice. The period of the lattice in $z$ direction is equal $2 s$ which is much less than the period along the $y$ axis $a=\varphi_{0} / B_{\|} s \ll \lambda_{J}$. The interaction between pancakes and in-plane field in the form of JVs is known to produce zigzag deformation of PV stacks [see Fig. 1(c)]. ${ }^{15,20,30}$ The zigzag deformation is somewhat larger near the surface (due to the decrease of the stifness of the PVs stack) but for simplicity we neglect this relatively small effect. The amplitude of the zigzag deformation

$$
\left|u_{n}\right|=u \simeq \frac{\lambda_{a b}}{\beta \ln \beta}, \quad \beta=\frac{B_{||} \lambda_{J}}{H_{0} \lambda_{a b}} \gg 1,
$$

is assumed to be the same for all $N$ layers and corresponds to the equilibrium form of the zigzag vortex in a bulk $(N \rightarrow \infty)$ layered superconductor. ${ }^{9,30}$ In this case the centers of pancakes for both vortex lines are positioned at a zigzag line

$$
\mathbf{r}_{n}^{(1)}=(-1)^{n}{ }^{1} u \mathbf{x}_{0} \quad \mathbf{r}_{n}^{(2)}=\mathbf{r}_{n}^{(1)}+\mathbf{R},
$$

and the Eq. (8) with respect to functions $f_{n}(\mathbf{q})$ looks differently for odd and even $n$. Introducing two new functions $f_{1 k}$ (q) and $f_{2 k}(\mathbf{q})$

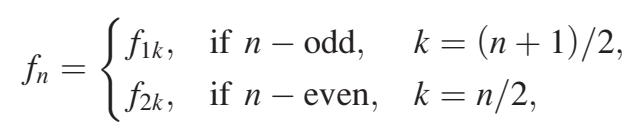

Eq. (8) can be rewrited as follows:

$$
\begin{aligned}
f_{1,2 k} & +\frac{1}{2 q \Lambda} \sum_{m} \mathrm{e}^{2|k m| q s} f_{1,2 m}+\frac{1}{2 q \Lambda} \sum_{m} \mathrm{e}^{(2|k m|+1) q s} f_{2,1 m} \\
= & \mathrm{e}^{ \pm i q_{x} u} .
\end{aligned}
$$

The interaction energy (11) expressed in terms of the scalar functions $f_{1 k}(\mathbf{q})$ and $f_{2 k}(\mathbf{q})$ reads

$$
\begin{aligned}
\varepsilon_{\text {int }}(\mathbf{R})= & \frac{\varphi_{0}^{2}}{16 \pi^{3} \Lambda} \int \frac{d^{2} \mathbf{q}}{q^{2}} \cos (\mathbf{q} \mathbf{R}) \\
& \times \sum_{k}^{N}\left[f_{1 k}(\mathbf{q}) e^{i q_{x} u}+f_{2 k}(\mathbf{q}) e^{i q_{x} u}\right] .
\end{aligned}
$$

The expression (21) and the system of algebraic equation (20) determine the interaction energy of two identical zigzag vortex lines which are shifted at the vector $\mathbf{R}$ in the $x y$ plane.

For the case of a large intervortex distance $R \gg s, u$, we may use the continuous limit for the solution of the problem (20) because for the relevant wave vectors $q s \ll 1$ and $q_{x} u$ $\ll 1$. We introduce a continuous coordinate $z=2 \mathrm{ks}$ and continuous functions $f_{1,2}(\mathbf{q}, z)$ defined at the interval $|z| \leq D / 2$, where $D$ is the total thickness of superconducting film. Thus, the linear system of Eqs. (20) reduces to the following integral equations:

$$
\begin{aligned}
& f_{1,2}(q, z)+\frac{1}{4 q \Lambda s} \int_{D / 2}^{D / 2} d z^{\prime} \mathrm{e}^{q \mid z} z^{\prime} \mid f_{1,2}\left(q, z^{\prime}\right) \\
& +\frac{\mathrm{e}^{q s}}{4 q \Lambda s} \int_{D / 2}^{D / 2} d z^{\prime} \mathrm{e}^{q \mid z} z^{\prime} \mid f_{2,1}\left(q, z^{\prime}\right)=\mathrm{e}^{ \pm i q_{x} u} .
\end{aligned}
$$

Using the solutions of Eq. (22), we may rewrite the expression for interaction energy (21) as follows (see Ref. 34 for details):

$$
\begin{aligned}
\varepsilon_{\text {int }}(\mathbf{R})= & \frac{\varphi_{0}^{2}}{16 \pi^{3} \Lambda s} \int d^{2} \mathbf{q} \cos (\mathbf{q R}) \\
& \times\left\{D\left[\frac{\cos ^{2}\left(q_{x} u\right)}{p_{+}^{2}}+\frac{\sin ^{2}\left(q_{x} u\right)}{q^{2}}\right]\right. \\
& \left.+\frac{2 q \cos ^{2}\left(q_{x} u\right)}{\lambda_{a b}^{2} p_{+}^{4}\left(1+q / p_{+} \tanh L_{+}\right)}\right\},
\end{aligned}
$$

where

$$
p_{+}^{2} \simeq q^{2}+1 / \lambda_{a b}^{2}, \quad p^{2} \simeq q^{2}, \quad L_{ \pm}=p_{ \pm} D / 2 .
$$

Performing in (23) the necessary integration, we finally obtain the following expression for the interaction energy of two identical zigzag pancake stacks which are shifted at the vector $\mathbf{R}=R \mathbf{x}_{0}$ in the $x y$ plane

$$
\begin{aligned}
& \varepsilon_{\text {int }}(R)=\frac{\varphi_{0}^{2}}{32 \pi^{2} \Lambda s}\left\{D\left[2 K_{0}\left(\frac{R}{\lambda_{a b}}\right)+K_{0}\left(\frac{R-2 u}{\lambda_{a b}}\right)+K_{0}\left(\frac{R+2 u}{\lambda_{a b}}\right)+\ln \left(\frac{R^{2}-4 u^{2}}{R^{2}}\right)\right]\right. \\
& \left.+\frac{2}{\lambda_{a b}^{2}} \int_{0}^{\infty} \frac{d q}{\left(q^{2}+\lambda_{a b}^{2}\right)^{2}} \frac{2 J_{0}(q R)+J_{0}(q(R-2 u))+J_{0}(q(R+2 u))}{1+q / \sqrt{q^{2}+\lambda_{a b}^{2} \tanh 1\left(D \sqrt{q^{2}+\lambda_{a b}^{2} / 2}\right)}}\right\}
\end{aligned}
$$

where $J_{0}$ and $K_{0}$ are the Bessel and modified Bessel functions of zero order, respectively. The first term proportional to $D$ in Eq. (25) describes the interaction between the zigzag stacks in the bulk layered system, ${ }^{9}$ while the last term is responsible for the influence of film boundaries. In Fig. 3 we present some typical plots of the interaction energy per one layer $\varepsilon_{\text {int }} / N$ vs the intervortex distance $R$ for different thick5 


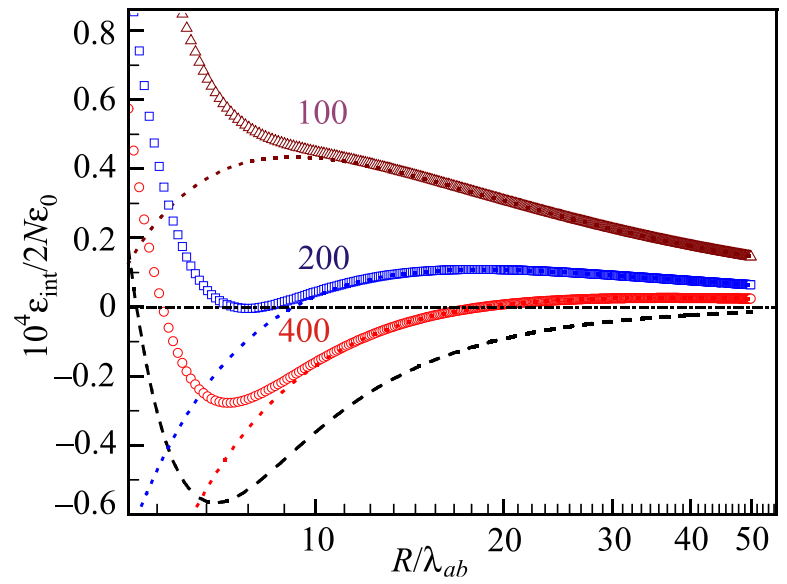

Fig. 3. Typical plots of the interaction energy per layer $\varepsilon_{\text {int }}(R) / 2 N \varepsilon_{0}$ [Eq. (25)] vs the distance $R$ between two zigzag deformed stacks of pancakes for in plane magnetic field $B_{\|} / H_{0} \quad 1$. The numbers near the curves denote the values of film thickness $D / \lambda_{a b}$. The dashed line shows the interaction energy between two zigzag vortices in bulk $(D \rightarrow \infty)$ layered SC. Dotted lines show the long range part of interaction energy (26). Here $\varepsilon_{0} \quad \varphi_{0}^{2} / 32 \pi^{2} \lambda_{a b}$, s $\quad 0.01 \lambda_{a b}, \gamma \quad 300$.

At long distances $R \gg \lambda_{a b}$, the modified Bessel functions decay exponentially, and a bulk short-range repulsion between two PVs stacks is negligible small. The leading bulk contribution comes from the logarithmic term in (25), which describes an attraction between the zigzag PV lines. The last term in (25) describes long-range Pearl's repulsion which decays as $1 / R$ and results from the surface contribution to the energy. Assuming that the effect of the zigzag deformation $u \ll \lambda_{a b}$ is small, the long-range part of interaction energy (25) for $R$ $\gg \lambda_{a b}$ can be written as

$$
\varepsilon_{\text {int }}(R) \simeq \frac{\varphi_{0}^{2}}{8 \pi^{2}}\left[-\frac{D u^{2}}{\lambda_{a b}^{2} R^{2}}+\frac{2}{R}+\frac{4 u^{2}}{R^{3}}\right] .
$$

One can observe an interplay of the long-range attractive and the repulsive forces between two zigzag deformed PV stacks, similar to the one between two tilted vortices in anisotropic SC films. ${ }^{24,28}$ The last term in (26) describes a modification of long-range Pearl's repulsion due to zigzag deformation of the PVs stack. Certainly, in a bulk sample $(D \rightarrow \infty)$ the Pearl's term in (26) vanishes, and at long distances the dominant interaction between the zigzag PV lines is an attraction. ${ }^{9}$ As a result, in a bulk sample there always exists the minimum of the interaction potential, which realizes with logarithmic accuracy at $R_{m} \approx 2 \lambda_{a b} \ln \left(\lambda_{a b} / u\right)$. However the second term in (26) is very important even for a rather thick films: for large $R$ the energy $\varepsilon_{\text {int }}$ is always positive and corresponds to the vortex repulsion. With a decrease of the distance $R$ the attraction force comes into play and can result in the change of the sign of the energy at $R_{0} \approx D u^{2} / 2 \lambda_{a b}^{2} \gg \lambda_{a b}$, while shortrange repulsion is still weak. Such behavior should be accompanied by the appearance of the minimum in the interaction potential. The condition $R_{0}>R_{m}$ gives us a rough estimate of the critical film thickness $D_{\text {cr }}$ for which the minimum in the interaction potential can exist and the formation of vortex chains can be energetically favorable:

$$
D_{\mathrm{cr}} \approx 4 \sigma \lambda_{a b} \beta^{2}(\ln \beta)^{3} \sim \frac{4 \sigma \lambda_{J}^{2}}{\lambda_{a b}}\left(\frac{B_{\|}}{H_{0}}\right)^{2},
$$

where the constant $\sigma$ is of the order unity. The more accurate criterion of attraction of zigzag PVs stacks should be based on the expression for the interaction energy (25) and may be found from the conditions

$$
\varepsilon_{\text {int }}(R)=\varepsilon_{\text {int }}^{\prime}(R)=0 .
$$

The typical dependence of the critical film thickness $D_{\text {cr }}$ as a function of in-plane magnetic field $B_{\|}$is shown in Fig. 4. So, in the presence of a dense lattice of Josephson vortices, the AVs penetrate in the form of chains only for a large film thickness $D>D_{\mathrm{cr}}$. Otherwise, if $D<D_{\mathrm{cr}}$, the formation of the usual Abrikosov lattice of zigzag deformed stacks of PVs occurs. It is interesting to note that following (26) the intervortex attraction increases near the critical temperature of the superconducting transition $T_{c}$, when $\lambda_{a b}$ becomes large (except for the region very close to $T_{c}$ where the condition $\lambda_{a b} \ll \lambda_{J}$ is not satisfied). This behavior is in contrast with the moderately anisotropic case ${ }^{28}$ when the critical thickness for intervortex attraction to be observed decreases near $T_{c}$ and the repulsion between vortices prevails.

\subsection{Interaction potential of two stacks in the crossing lattices}

In the high in-plane field limit $B_{\|} \gg H_{0}$ considered in Sec. 2.3 the triangular lattice of JVs is so dense that the currents of adjacent JVs ovrlap setrongly and the amplitude of the zigzag deformation decreases. ${ }^{30}$ As a result, the critical thickness $D_{\text {cr }}$ grows rapidly with an increase of the in-plane field $B_{\|}$(see Fig. 4). Also the potential dip in the intervortex interaction energy (25) strongly decreases with an increase of $B_{\|}{ }^{9}$. Optimal regime for the long-ranged attraction corresponds to $B_{\|} \sim H_{0}$. If the magnetic field $B_{\|}$is small, the crossings between JVs and PVs are rare and the distance between Josephson vortices along the $z$ axis becomes much larger than $2 s$, as well as the distance between the deformed parts of the PVs line [see Fig. 1(b)]. Let us estimate the attraction between two deformed stacks in this limit. The Josephson vortices are well separated and positioned at the distance

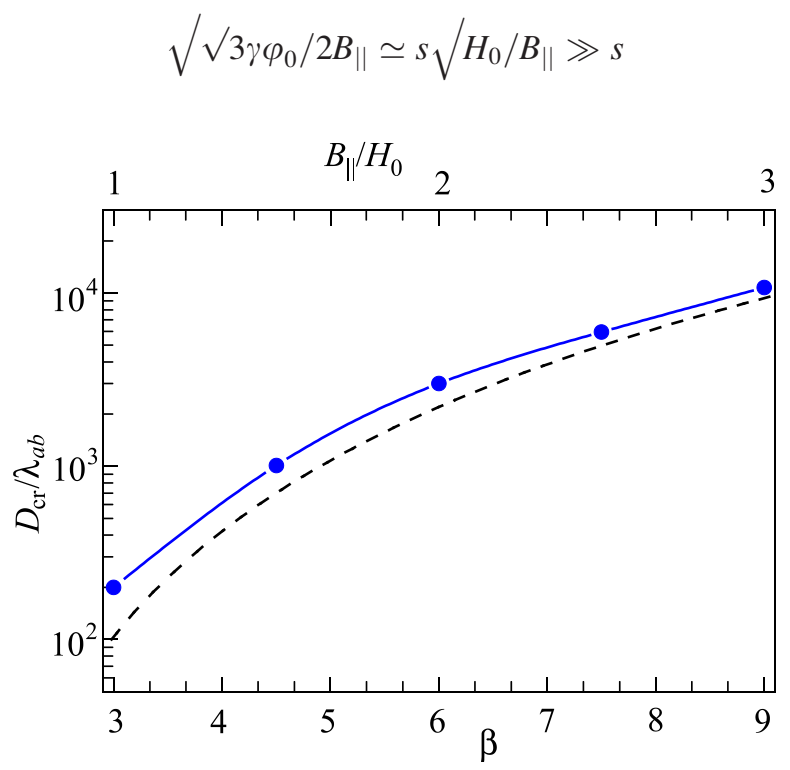

Fig. 4. Critical film thickness $D_{\mathrm{cr}}$ as a function of in plane magnetic field $B_{\|}$ ( - ). The dashed line shows the estimate of the critical film thickness (27). Here $\beta \quad B_{\|} \lambda_{J} / H_{0} \lambda_{a b}, s \quad 0.01 \lambda_{a b}, \gamma \quad 300, \lambda_{J} \quad 3 \lambda_{a b}, \sigma 2$. 
along the $z$ axis. As has been demonstrated in Ref. 9, the contribution to the attraction from one crossing is

$$
\tilde{\varepsilon}_{\text {att }}(R)=-\frac{s \varphi_{0}^{2}}{R^{2}}\left[\frac{\lambda_{a b}}{\lambda_{J} \ln \left(A \lambda_{J} / \lambda_{a b}\right)}\right]^{2},
$$

where $A \approx 3.5$ (see Ref. 15 ). Neglecting the effect of a zigzag deformation on the Pearl's repulsion, the long-ranged part of interaction energy $\tilde{\text { int }}(R)$ in low-field limit $B_{\|} \ll H_{0}$ at distances $R \gg \lambda_{a b}$ may be estimated as

$$
\tilde{\varepsilon}_{\text {int }}(R) \simeq \frac{\varphi_{0}^{2}}{8 \pi^{2}}\left[-\frac{D \tilde{u}^{2}}{\lambda_{a b}^{2} R^{2}}+\frac{2}{R}\right],
$$

where the effective displacement ${ }^{9,15}$

$$
\tilde{u} \simeq \frac{2 \sqrt{ } 2 \lambda_{a b}^{2}}{\lambda_{J} \ln \left(A \lambda_{J} / \lambda_{a b}\right)}\left(\frac{B_{\|}}{H_{0}}\right)^{1 / 4}
$$

grows slowly with increasing in-plane magnetic field $B_{\|}$. The expressions (29), (30) result in the following estimate of the critical film thickness $\tilde{D}_{\text {cr }}$ :

$$
\tilde{D}_{\mathrm{cr}} \sim \frac{\lambda_{J}^{2} \ln ^{2}\left(A \lambda_{J} / \lambda_{a b}\right)}{2 \lambda_{a b}}\left(\frac{B_{\|}}{H_{0}}\right)^{1 / 2} .
$$

As before, the case $B_{\|} \sim H_{0}$ is optimal for observation of intervortex attraction and this case corresponds to the crossover between the regimes described by formulas (27) and (31). For typical experimental value of the in-plane magnetic field ${ }^{17} B_{\|}=2030 \mathrm{G}$ we obtain $\tilde{D}_{\text {cr }} \sim 70 \mu \mathrm{m}$.

Thus, the relations (27), (31) give us the estimate of the threshold value of the thickness

$$
D> \begin{cases}\tilde{D}_{\mathrm{cr}}, & \text { if } B_{\|}<H_{0} \\ D_{\mathrm{cr}}, & \text { if } B_{\|}>H_{0}\end{cases}
$$

for which the minimum in the interaction potential can exist and the interplay between the long-range attraction and repulsion between zigzag vortex stacks lines in the films of layered superconductors takes place. We should note that the Pearl's interaction plays an important role even for rather thick film. Indeed, for the most favorable case $B_{\|} \sim H_{0}$ this interaction completely masks the attraction for the film thicknesses smaller than $\sim 50200 \lambda_{a b}$.

\section{Vortex molecules}

The vortex attraction in bulk layered superconductors is known to result in the formation of infinite vortex chains. But even in the regime when the intervortex attraction exists, the formation of infinite chains can be questioned for rather thin films. The point is that, despite the fact that two vortices attract each other, further increase in the number of vortices arranged in a chain can be energetically unfavorable because of the slower decay of the long-ranged Pearl's repulsive force compared to the attractive one. Therefore, for rather thin samples, there appears an intriguing possibility to observe vortex chains of finite length, i.e., vortex molecules or clusters. The calculations ${ }^{24,28}$ confirm this and indeed the number of vortices energetically favorable in a molecule grows as we increase the film thickness and/or the tilting angle because of the increasing attraction term in the pair potential $\varepsilon_{\text {int }}(15)$ and (16). In Ref. 28 the intervortex interaction has been also calculated and analyzed taking account of the equilibrium shape of tilted vortex lines.

Figure 5 shows schematic pictures of vortex matter consisting of dimeric [Fig. 5(a)] and trimeric [Fig. 5(b)] molecules, indicating that the vortex lattices contain more than one vortex per unit cell. The transitions between different multiquanta vortex lattices should occur with the change of the tilting angle and field strength. ${ }^{28}$ Finally, for rather thick samples with $d \gg \lambda_{a b}$ we get a standard infinite chain structure typical of bulk systems [Fig. 5(c)]. Note that the formation of an infinite vortex chain may be considered in some sense as a polymerization of the vortex molecules. Certainly, the crossover from the vortex molecule state to the infinite chain structure is strongly influenced by the increase in the vortex concentration governed by the component of the external magnetic field perpendicular to the film. Indeed, one can expect such a crossover to occur when the mean intervortex spacing approaches the molecular size.

The experiments ${ }^{24}$ were performed at constant orientation of the applied magnetic field. Therefore, by varying the magnetic field, changes of both the tilting angle and vortex concentration were realized. As the vortex concentration was relatively high, we could not expect to observe the molecules (in this regime the average distance between vortices must be much larger than the size of the molecule). To observe the vortex molecules (or multiquanta vortex lattices) it would be preferable to change only the parallel component of the magnetic field, by varying the vortex tilting angle while not affecting the vortex concentration, which must be very low to avoid the inter-molecule interaction.
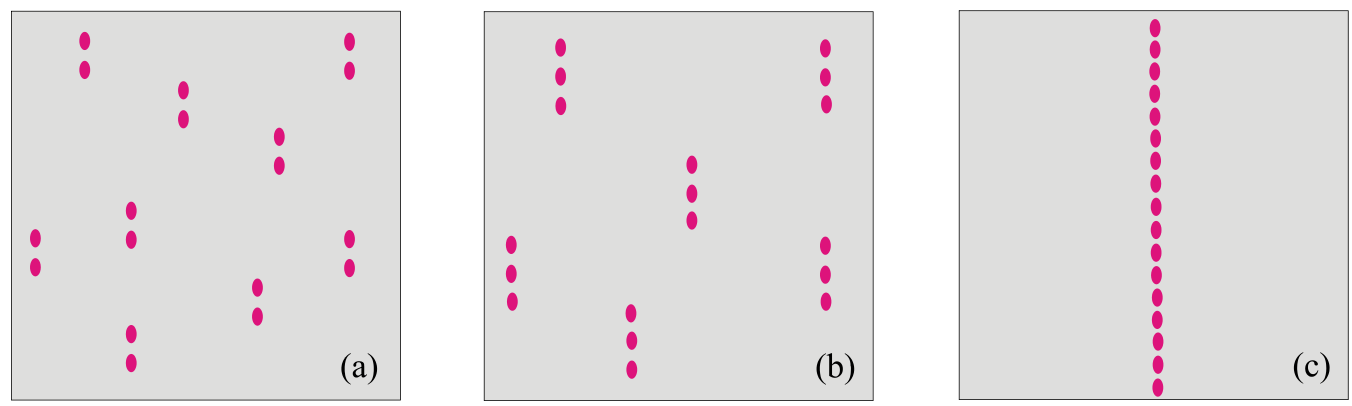

Fig. 5. Schematic pictures of vortex matter consisting of dimeric molecules (a), trimeric molecules (b) and infinite vortex chain (c). Vortex positions are denoted by filled ellipses. 
Qualitatively all conclusions of Refs. 24 and 28 are applicable for the case of crossing lattices. Similarly, the zigzags of PVs stacks can be arranged in vortex molecules. To estimate the size of such molecule let's find the cohesion energy of equidistant chain of deformed PVs stacks. The perpendicular component of the magnetic field $B_{\perp}$ fixes the number $M$ of AVs in the film, so that in the square film $W \times W$ it should be $M=B_{\perp} W^{2} / \varphi_{0}$. vortices. We consider the case of small concentration of vortices when at large distances the intervortex interaction is given by Pearl's term. For roughly uniform vortex distribution (usual vortex lattice) the energy per one vortex can be estimated as $\varepsilon_{0} \sim \varphi_{0} B_{\perp} W / 4 \pi^{2}$. If vortices form clusters with $m$ vortices each, it may be demonstrated that interaction between clusters gives the same contribution $\varepsilon_{0}$ per one vortex. The remaining energy originates from the interaction between vortices inside cluster, and for the case of a low in-plane magnetic field $B_{\|} \ll H_{0}$, it may be expressed via the long-ranged part of interaction energy $\tilde{\varepsilon}_{\text {int }}(29)$

$$
\varepsilon_{\text {int }}^{m}=\frac{1}{m} \sum_{i>j}^{m} \tilde{\varepsilon}_{\text {int }}\left(R_{i j}\right),
$$

where $R_{i j}$ are the distances between $i$ th and $j$ th vortices in the chain molecule. Taking the characteristic distance between vortices in a cluster $\tilde{R}_{m} \approx 2 \lambda_{a b} \ln \left(\lambda_{J} / \lambda_{a b}\right)$ we find

$$
\varepsilon_{\text {int }}^{m} \sim \frac{\varphi_{0}^{2}}{8 \pi^{2}}\left[-\frac{D \tilde{u}^{2}}{\lambda_{a b}^{2} \tilde{R}_{m}^{2}}+\frac{2}{\tilde{R}_{m}} \ln m\right] .
$$

Certainly the cluster formation occurs if $\varepsilon_{\text {int }}^{m}<0$ and the number of vortices in the cluster is given by the expression

$$
\left.m \approx \exp \frac{D \tilde{u}^{2}}{2 \lambda_{a b}^{2} \tilde{R}_{m}}\right)
$$

The condition $m>1\left(D \tilde{u}^{2} / 2 \lambda_{a b}^{2} \tilde{R}_{m}>1\right)$ gives us the low boundary of the in-plane magnetic field restricting the interval of vortex molecules existence

$$
\frac{B_{\|}}{H_{0}}>\left[\frac{\lambda_{J}^{2} \ln ^{3}\left(\lambda_{J} / \lambda_{a b}\right)}{2 D \lambda_{a b}}\right]^{2}
$$

For $D=100 \lambda_{a b}, \lambda_{J}=3 \lambda_{a b}$ and $H_{0}=3 \mathrm{~T}$ we obtain $B_{\|}>60 \mathrm{G}$. Note that the number of vortices $m$ in the molecules rapidly increases as the in-plane magnetic field $B_{\|}$grows.

Naturally this scenario realizes only for low concentration of AVs when the average distance between vortices $\sqrt{ } \varphi_{0} / B_{\perp}$ is much larger than the intervortex distance in the chain $\tilde{R}_{m}$. In the opposite case we may expect the existence of multiquanta flux lattice with several vortices per cell similar to the case of tilted vortices considered in Ref. 28.

\section{Conclusions}

To sum up, we have analyzed some distinctive features of the vortex matter in thin films of layered superconductors focusing mainly on the effects caused by the tilted magnetic field. The interplay of electrodynamic interactions inside and outside the film results in a very peculiar behavior of the intervortex interaction potential which includes both attractive and repulsive terms. This interplay can change essentially the structural properties of the vortex matter which become quite different from the ones suggested in the original work by Abrikosov. In particular, we predict the possible formation of vortex clusters or molecules which can be also viewed as the multiplication of the unit cell for a regular vortex array.

This work was supported, in part, by the Russian Foundation for Basic Research, the EU COST Action CA16218, the French ANR project "Optofluxonics" (AIB) and Foundation for the Advancement of Theoretical Physics "Basis" (ASM). The numerical simulations were carried out with the support of the Russian Science Foundation (Project No. 17-12-01383). AIB thanks the Leverhulme Trust for supporting his stay in Cambridge 768 University.

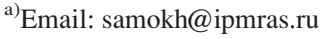

${ }^{1}$ G. Blatter, M. V. Feigel'man, V. B. Geshkenbein, A. I. Larkin, and V. M. Vinokuret, Rev. Mod. Phys. 66, 1125 (1994).

${ }^{2}$ P. G. De Gennes, Superconductivity of Metals and Alloys (Benjamin, New York, 1966).

${ }^{3}$ A. A. Abrikosov, Fundamentals of the Theory of Metals (North Holland, Amsterdam, 1988).

${ }^{4}$ A. I. Buzdin and A. Yu. Simonov, Pis'ma Zh. Eksp. Teor. Fiz. 51, 168 (1990) [JETP Lett. 51, 191 (1990)].

${ }^{5}$ A. I. Buzdin and A. Yu. Simonov, Physica C 168, 421 (1990).

${ }^{6}$ A. M. Grishin, A. Yu. Martynovich, and S. V. Yampolskii, Sov. Phys. JETP 70, 1089 (1990) [Zh. Eksp. Teor. Fiz. 97, 1930 (1990)].

${ }^{7}$ V. G. Kogan, N. Nakagawa, and S. L. Thiemann, Phys. Rev. B 42, 2631 (1990).

${ }^{8}$ A. I. Buzdin and A. Yu. Simonov, Physica C 175, 143 (1991).

${ }^{9}$ A. Buzdin and I. Baladie, Phys. Rev. Lett. 88, 147002 (2002).

${ }^{10}$ M. J. W. Dodgson, Phys. Rev. B 66, 014509 (2002).

${ }^{11}$ P. L. Gammel, D. J. Bishop, J. P. Rice, and D. M. Ginsberg, Phys. Rev. Lett. 68, 3343 (1992).

${ }^{12}$ H. F. Hess, C. A. Murray, and J. V. Waszczak, Phys. Rev. Lett. 69, 2138 (1992).

${ }^{13}$ A. Tonomura, H. Kasai, O. Kamimura, T. Matsuda, K. Harada, T. Yoshida, T. Akashi, J. Shimoyama, K. Kishio, T. Hanaguri, K. Kitazawa, T. Masui, S. Tajima, N. Koshizuka, P. L. Gammel, D. Bishop, M. Sasase, and S. Okayasu, Phys. Rev. Lett. 88, 237001 (2002).

${ }^{14}$ S. J. Bending and M. J. W. Dodgson, J. Phys.: Condens. Matter 17, R955 (2005).

${ }^{15}$ A. E. Koshelev, Phys. Rev. Lett. 83, 187 (1999).

${ }^{16}$ L. N. Bulaevskii, M. Ledvij, and V. G. Kogan, Phys. Rev. B 46, 366 (1992).

${ }^{17}$ A. Grigorenko, S. Bending, T. Tamegai, S. Ooi, and M. Henini, Nature (London) 414, 728 (2001).

${ }^{18}$ C. A. Bolle, P. L. Gammel, D. G. Grier, C. A. Murray, D. J. Bishop, D. B. Mitzi, and A. Kapitulnik, Phys. Rev. Lett. 66, 112 (1991); I. V. Grigorieva, J. W. Steeds, G. Balakrishnan, and D. M. Paul, Phys. Rev. B 51, 3765 (1995); M. Tokunaga, T. Tamegai, Y. Fasano, and F. de la Cruz, ibid. 67, 134501 (2003)

${ }^{19}$ V. K. Vlasko Vlasov, A. Koshelev, U. Welp, G. W. Crabtree, and K. Kadowaki, Phys. Rev. B 66, 014523 (2002).

${ }^{20}$ A. E. Koshelev, Phys. Rev. B 71, 174507 (2005).

${ }^{21}$ J. Pearl, Appl. Phys. Lett. 5, 65 (1964).

${ }^{22}$ A. Tonomura, Electronic Holography, 2nd ed., Springer Series in Optical Sciences Vol. 70 (Springer, Berlin, Heidelberg, 1999).

${ }^{23}$ K. Harada, T. Matsuda, J. Bonevich, M. Igarashi, S. Kondo, G. Pozzi, U. Kawabe, and A. Tonomura, Nature 360, 51 (1992).

${ }^{24}$ A. I. Buzdin, A. S. Mel'nikov, A. V. Samokhvalov, T. Akashi, T. Masui, T. Matsuda, S. Tajima, H. Tadatomo, and A. Tonomura, Phys. Rev. B 79, 094510 (2009).

${ }^{25}$ W. E. Lowrence and S. Doniach, in Proceedings of 12th International Conference on Low Temperature Physics, Kyoto, edited by E. Kanda (1970), p. 361.

${ }^{26}$ A. I. Buzdin and D. Feinberg, J. Phys. (Paris) 51, 1971 (1990); J. R. Clem, Phys. Rev. B 43, 7837 (1991); K. Efetov, Sov. Phys. JETP 49, 905 (1979); S. N. Artemenko and A. N. Kruglov, Phys. Lett. A 143, 485 (1990).

${ }^{27}$ L. Bulaevskii and J. R. Clem, Phys. Rev. B 44, 10234 (1991). 
${ }^{28}$ A. V. Samokhvalov, D. A. Savinov, A. S. Mel'nikov, and A. I. Buzdin, Phys. Rev. B 82, 104511 (2010).

${ }^{29}$ J. Pearl, J. Appl. Phys. 37, 4139 (1966).

${ }^{30}$ L. N. Bulaevskii, M. Maley, H. Safar, and D. Dominguez, Phys. Rev. B 53, 6634 (1996).

${ }^{31}$ J. Mirkovic, S. E. Savel'ev, E. Sugahara, and K. Kadowaki, Phys. Rev. Lett. 86, 886 (2001); 66, 132505 (2002); M. Konczykowski, C. J. van der Beek, A. E. Koshelev, V. Mosser, M. Dodgson, and P. H. Kes, Phys. Rev. Lett. 97, 237005 (2006).
${ }^{32}$ V. Pudikov, Physica C 212, 155 (1993).

${ }^{33}$ T. Pe, M. Benkraouda, and J. R. Clem, Phys. Rev. B 55, 6636 (1997).

${ }^{34}$ A. V. Samokhvalov, A. S. Mel'nikov, and A. I. Buzdin, Phys. Rev. B 85, 184509 (2012). 